\title{
Sequencing Algorithm with Multiple-Input Genetic Operators: Application to Disaster Resilience
}

\author{
Aman Karamlou, Paolo Bocchini* \\ Department of Civil and Environmental Engineering, ATLSS Engineering Research \\ Center, Lehigh University, 117 ATLSS Drive, Bethlehem, PA 18015-4729, USA
}

\begin{abstract}
A novel evolutionary optimization methodology called "Algorithm with MultipleInput Genetic Operators" (AMIGO) for scheduling independent tasks considering resource and time constraints is presented. $A M I G O$ is characterized by new genetic operators enriched with complementary information, including auxiliary variables computed by the fitness function, as well as the global parameters of the problem. The application of $A M I G O$ to multi-phase optimal resilience restoration scheduling of highway bridges is presented and discussed. To this purpose, enhancements have been made also to the bridge network resilience analysis (a new performance metric and restoration model). The quality of the solution and efficiency of $A M I G O$ are demonstrated through the application to a large transportation network subjected to earthquake.
\end{abstract}

\footnotetext{
${ }^{*}$ Corresponding author. Phone: +1 610-758-3066.

Email addresses: amk211@lehigh . edu (Aman Karamlou), paolo.bocchini@lehigh .edu (Paolo Bocchini)
} 


\section{Introduction}

This paper presents a new multiple-objective optimization methodology, consisting in an evolutionary solution technique for scheduling of independent tasks, considering time and logistic constraints. The optimization solution methodology

5 called Algorithm with Multiple-Input Genetic Operators (AMIGO) includes novel genetic operators, which take advantage of auxiliary variables computed during the fitness evaluation process, as well as some global problem parameters, to enhance the search procedure. Figure 1 highlights this feature compared to common genetic algorithms. The versatility of the proposed methodology makes it applicable to a variety of scheduling problems common in different fields, such as construction management, production and manufacturing industry, and emergency planning. There has been an extensive research in the field of project scheduling optimization. Alternative approaches and their foundations have been discussed in [1-4]. Also, extensive review of different techniques and formulations of project scheduling problems can be found in [5-8]. In many classical forms of project management and scheduling, the objective of the problem can be formulated in closed-form, such as project duration, time lag, lateness, and resource consumption [1-4]. However, in the majority of real-world applications, the objective functions of scheduling optimization problems cannot be presented by an algebraic model due to the complexity involved in the computation of the objectives. Focusing on such cases, the heuristic nature of $A M I G O$, eliminates the constraints on the form of the objective, and therefore can be used for any type of function. An effort has been made to make the technique appealing for practical applications, in terms of simplicity, efficiency, and applicability to large real-word problems. 
covery is presented. In particular, the prompt recovery of infrastructure systems is pursued. In fact, the life of the modern society is highly reliant on its critical lifelines and infrastructures, such as healthcare facilities, water and electric supplies, and transportation networks. In the case of an extreme event (such as earthquake or hurricane), the long lasting loss of functionality of these vital elements contributes considerably to the reduction of habitability of the impacted regions. The consequent gradual (large-scale) migration of the population is one of the major threats to the community's recovery process and its social and economic integrity [9, 10]. Therefore, it is imperative for the communities located in disaster prone regions to have a comprehensive pre-disaster mitigation and post-event restoration plan, which allows to retrofit the most vulnerable components, and to recover efficiently from the unavoidable damage. This encouraged researchers from several academic fields, as well as disaster officials, to pay a paramount attention to the topics of disaster mitigation, emergency management, and restoration planning. As a result, several planning and scheduling computational models and optimization methodologies have been proposed and developed for a variety of applications. Along this line of research, $A M I G O$ is a major improvement in the ability to consider very complex objectives, while maintaining the computational cost acceptable for realistic applications.

In particular, in this paper $A M I G O$ is applied to the post-disaster repair and restoration prioritization of damaged highway networks. Among various infrastructure systems, transportation networks require particular attention, as the performance and quality of services provided by many other physical and socioeconomic units (e.g., businesses) are highly dependent on the functionality of transportation networks, both in ordinary and emergency situations. The func- 
tionality of transportation networks is threatened by different factors (e.g., closure of roads due to collapse-induced debris or landslides), among which damage to the bridges is the most common and critical. To this respect, AMIGO was tailored to find the optimal restoration sequence of the damaged bridges in the context of a highway network. Among several infrastructure system performance criteria, resilience has been selected as the objective of the optimization problem. Resilience assessment requires several sophisticated, iterative, and computationally expensive procedures. Moreover, these procedures can be formulated only algorithmically, thus the objective function cannot be coded in closed-form. These challenges make resilience assessment a perfect benchmark to test $A M I G O$ and showcase its characteristics.

The notion of resilience was first propounded by ecologists to describe the reorganizing capability of ecological systems after a disruption, to a new stable state [11, 12]. In the engineering world, resilience has been generally defined as the ability of social units to absorb, withstand, and efficiently recover from a perturbation [13]. Bruneau et al. [14] have first presented a conceptual framework for quantitative assessment of seismic resilience and highlighted the need for communities to focus on different aspects of this concept. Following this work, many researchers have presented procedures and metrics to quantify and enhance disaster resilience such as in [15-22]. Moreover, extensive studies have been performed on formulating and quantifying resilience for particular infrastructures and lifelines, such as healthcare facilities and hospitals [23], power grids [24], telecommunication systems [25], water supplies [26], and maritime transportation systems [27]. Regarding the recovery and resilience of transportation networks, Bocchini and 75 Frangopol [28, 29] presented a framework for quantifying the performance of 
highway networks considering the influence of the damaged bridges and optimizing the bridge restoration in terms of resilience and intervention cost. The impact of different retrofit techniques on seismic resilience of highway bridges has been presented in [30].

While the focus of this paper is the presentation and application of $A M I G O$, refinements have been performed also on the formulation of post-disaster resilience analysis of bridges and highway networks compared to the previous model presented in [31]. These are (1) a new bridge recovery model, and (2) the consideration of different phases of disaster management by introducing a new network connectivity-based resilience metric.

The performance of $A M I G O$ is demonstrated through a large-scale numerical example. The highway network serving the port of San Diego has been chosen for this purpose, which contains 238 highway bridges. The proposed methodology has been utilized to find the best restoration strategies for the bridges of the network damaged by an earthquake scenario, selected based on the seismicity of the region. In addition, the efficiency of the proposed formulation has been examined through comparison of the results with previous methodologies using smaller test networks.

\section{Proposed optimization technique: $A M I G O$}

In this section, the framework and solution strategy of the proposed scheduling optimization problem are presented in general terms. Several project scheduling problems have been addressed by the research community, considering different objectives (e.g., project duration, maximum activity lateness, net percent value), and constraints (e.g., precedence, resources, time lag). An extensive re- 

can be found in $[5-8]$. The optimization problem framework propounded in this study is a version of resource-constrained project scheduling with one renewable resource type, considering a maximum project duration and generic objectives. The problem is formulated as a combinatorial optimization characterized by a triple $\left(\mathbf{A}, N S A_{\max }, T\right)$. Activities (the words "activity" and "task" will be used interchangeably throughout this paper) of the project are collected in set $\mathbf{A}=\left\{A_{1}, A_{2}, \ldots, A_{n}\right\}$. To take into account logistic constraints (such as manpower, equipments, contractors) typically involved in planning, the number of simultaneous activities at each time step $t$ is limited to $N S A_{\max } . T$ is the investigated time span of the project. A schedule $\mathbf{S}=\left\{S_{1}, S_{2}, \ldots, S_{n}\right\}$ is defined as a vector in $\mathbb{R}^{n}$ such that $S_{i}$ is the start time of activity $A_{i}$. Finally, the quality of each schedule is determined by its associated fitness, which is a function of the sequence of tasks.

In many cases, the essential components (e.g., fitness functions and constraints) of the problem cannot be formulated in closed-form due to their complexity. This in fact, limits the application of standard optimization methodologies. For such cases, the advancement of heuristic optimization techniques, and evolutionary algorithms in particular, has resulted in high-quality near-optimal solutions for several challenging real-world optimization problems involving industrial, transportation, structural, and infrastructure engineering, among other fields.

$A M I G O$ belongs to this class of optimization solvers, which are powerful heuristic optimization techniques inspired by the process of natural selection and offer several advantages, such as applicability to almost any type of optimization problem (discrete and continuous), objective functions (differentiable and non- 
differentiable), and constraints (constrained and unconstrained domains). Moreover, they are particularly convenient for solving multi-objective optimization problems by Pareto-based approaches. Discussion about the fundamentals of evolutionary algorithms is beyond the scope of this paper and can be found for instance in [32]. The main characteristic of $A M I G O$ is the fact that it enriches the input of genetic operators with additional information on the outcome of the current individual, allowing for better convergence. Details on the characteristics and implementation of $A M I G O$ and its components including a new initial population generator and genetic operators are presented in the following subsections. For this study, $A M I G O$ and its components have been coded in the Matlab programming environment [33]. All subroutines are original, except for two operators (i.e. ranking and selection) extracted from the Elitist Non-Dominated Sorting Genetic Algorithms (NSGA-II) technique [34, 35]. Figure 2 shows the flowchart of $A M I G O$ as implemented in this study.

\subsection{Trial solution representation}

Let $\mathbf{A}$ be a set containing the $n$ activities of the project. The trial solution of the optimization problem is the array $\mathbf{x}^{\mathbf{s}}=\left\{x_{1}^{s}, x_{2}^{s}, \ldots, x_{n}^{s}\right\}$ such that $\mathbf{x}^{\mathbf{s}} \in P_{A}$, where $P_{A}$ is the space of all possible permutations of $\mathbf{A}$. Therefore, the trial solution is a sequence of tasks as shown in Figure $3 \mathrm{a}$. In cases where there is only one resource available (i.e. $N S A_{\max }=1$ ), the activities are simply performed in series.

Otherwise, if there are multiple resources (i.e. $N S A_{\max }>1$ ), the first $N S A_{\max }$ tasks are assigned to various resources, then, as soon as one activity is completed, the next task in array $\mathbf{x}^{\mathbf{s}}$ is assigned to the resource that has completed its job. In this process, it is assumed that the activities are performed consecutively, without any gap. 
The size of the design space seems to be equal to $n$ ! (i.e. all possible permutations of $n$ integers). However, there are several redundant solutions representing the same practical outcome. To avoid this redundancy, which would negatively impact the convergence of the evolutionary algorithm, the developed optimization operators interpret the individuals using two additional pieces of information: $N S A_{\max }$ and $l o^{s}$ (Figure 3 a). The former is a global parameter, whereas the latter is computed by the fitness function for each individual, as part of the assessment of the fitness itself. In many cases, not all activities can be completed during the specified time period $(T)$. In this respect, $l O^{s} \in \mathbf{x}^{\mathbf{s}}$ is defined as the last task in trial solution $\mathbf{x}^{\mathbf{s}}$ that starts and has an impact on the objective(s). With this representation, each trial solution is divided into three blocks, as shown in Figure 3b. All activities in block-1 start at the same time (i.e. $t=0$ ), then activities in block-2 are performed in sequence, and activities in block-3 are not performed in the investigated time. Obviously, the order of tasks appearing in blocks 1 and 3 is not important in determining the value of the objective(s). Therefore, the fitness of a solution depends only on the tasks that appear in the first block (regardless of their order, so a total number of $C_{N S A_{\max }}^{n}=\frac{n !}{N S A_{\max } !\left(n-N S A_{\max }\right) !}$ distinct cases), and the sequence of the tasks in the second block (for a total number of $\left(n-N S A_{\max }\right)$ ! permutations). Thus, the total number of unique solutions is actually only:

$$
C_{N S A_{\max }}^{n} \cdot\left(n-N S A_{\max }\right) !-\sum_{s=1}^{\frac{n !}{N S A_{\max } !}}\left[\left(n-l o^{s}\right) !-1\right]
$$

In Eq. (1), the second part (summation) excludes all possible permutations of the operations positioned after $l o^{s}$, for every solution $s$. 


\subsection{First generation}

A special subroutine has been developed with the aim of preventing the presence of redundant solutions in the first generation and enhance its quality with a preliminary optimization. To this purpose, block-1 of the individuals (Figure $3 \mathrm{~b}$ ) in the initial generation is obtained by randomly selecting one from all $C_{N S A_{\max }}^{n}$ possible sequences of tasks. Then, for each individual, the rest of the genes (i.e. block-2 and block-3) are filled by a randomly generated permutation of the remaining activities. It is worth mentioning that at this stage the fitness of the initial individuals is not yet evaluated. Therefore, the value of $l o^{s}$ for the solutions is not known and not used for the first generation.

In most optimization techniques, the quality of the search process, as well as the optimality of the final results can be improved considerably by selecting a good "starting point". In the case of the current problem, the first generation obtained by the presented procedure is mixed with the result of a customized "greedy algorithm" developed by the authors. The greedy algorithm starts considering all possible partial solutions of length $l_{\text {greedy }}^{0} \leq n$ and computes the values of the objective(s) considering the investigated $T$, assuming that only tasks present in the partial solution are accomplished. Then, the best of these solutions are extracted and their associated partial sequences are extended for the next step by adding all possible permutation of $\Delta l_{\text {greedy }}$ of the remaining tasks to the end of each solution. The value of $\Delta l_{\text {greedy }}$ is determined by the available computational capacity (e.g., 2). This process continues until the number of tasks in the optimal sequences reaches $\min \left(n, l o^{s}\right)$. Figure 4 illustrates the greedy algorithm procedure for the case of a project with 4 activities starting with partial solutions

of length $l_{\text {greedy }}^{0}=2$. The results obtained on several benchmark problems have 
shown that for small number of tasks the greedy algorithm finds some of the global optima, and for larger instances (e.g. $n>10$ ) it can enhance the convergence and reduces the overall computational cost.

\subsection{Multiple-input genetic operators}

The trial solution structure discussed in Section 2.1 is a "direct representation", meaning that the phenotype and genotype for the current problem are the same. Therefore, specialized genetic operators need to be developed to properly pass the important properties of the parents to the offspring [36]. The operators developed for $A M I G O$ take advantage of the two additional data $\left(N S A_{\max }, l o^{s}\right)$ shown in Figure 3, and search the design space more efficiently by not generating redundant trial solutions.

The novel mutation operator utilized by $A M I G O$ is a modified version of "swap mutation", which selects two genes randomly and swaps their contents [37]. The pseudo-code presented in Algorithm 1 shows the proposed mutation scheme. In particular, this procedure guarantees that no offspring is generated just by swapping two genes from block-1 or two genes from block-3.

Also a novel crossover operator has been developed for AMIGO, based on the well-known "order crossover" [37], which keeps a randomly selected array of subsequent genes from one parent, and fills the genes in the offspring by preserving their associated order in the other parent. In the developed crossover, first the common genes which appear in block-1 for both parents are passed to block-1 of the offspring. The same process is repeated on the common genes of the parents in block-3, which will be sent to the end of the offspring. Finally, the order crossover is carried out only on the remaining genes of the parents. The overall 


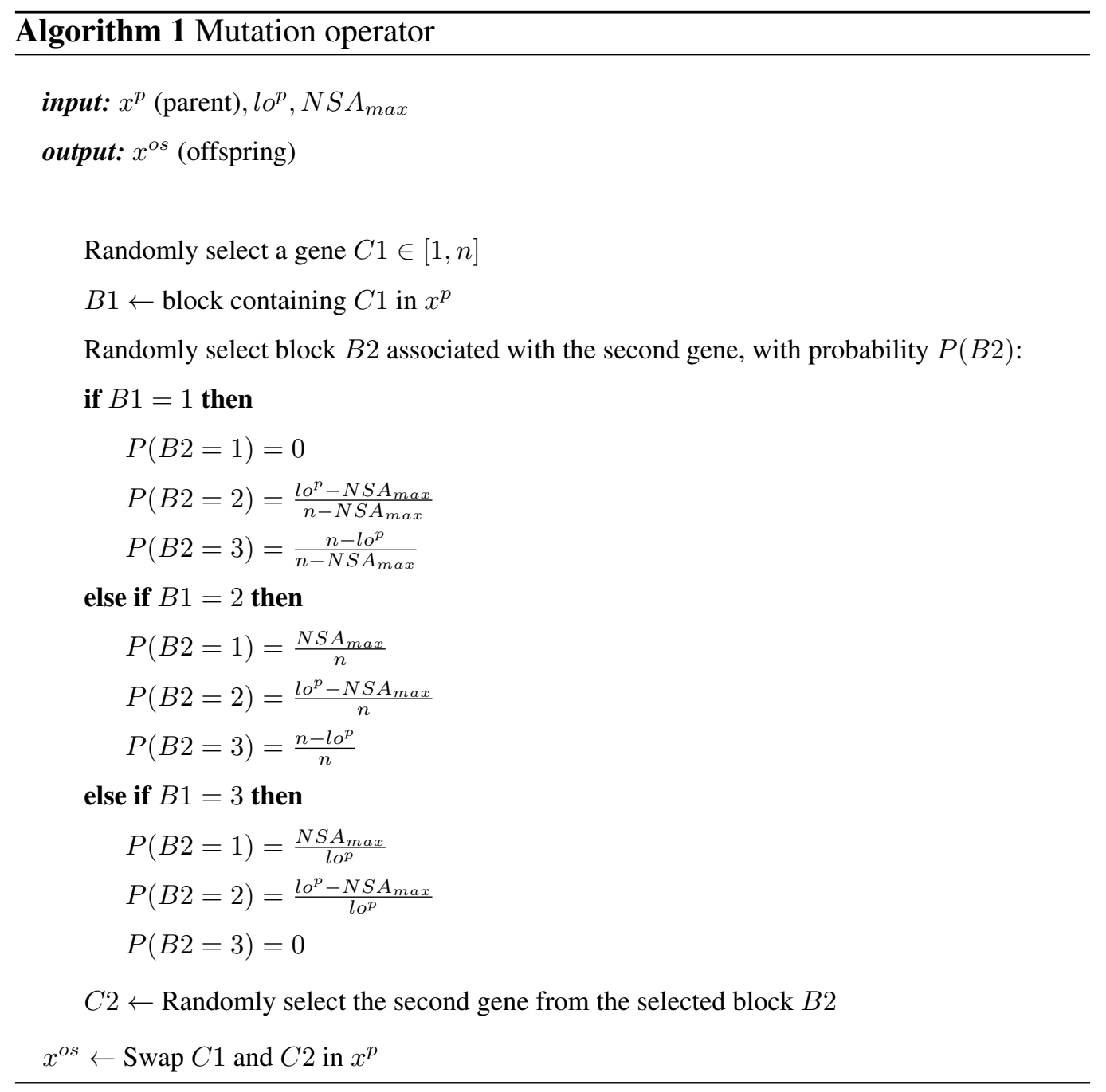

this crossover, the activities that are not performed by any parents have very low chance to be performed by the offspring. Also, the tasks that are carried out at the beginning of the process according to both parents receive higher priority in the offspring as well. Finally, the order crossover applied on the remaining activities will transfer some additional characteristics of the parents to the offspring. 


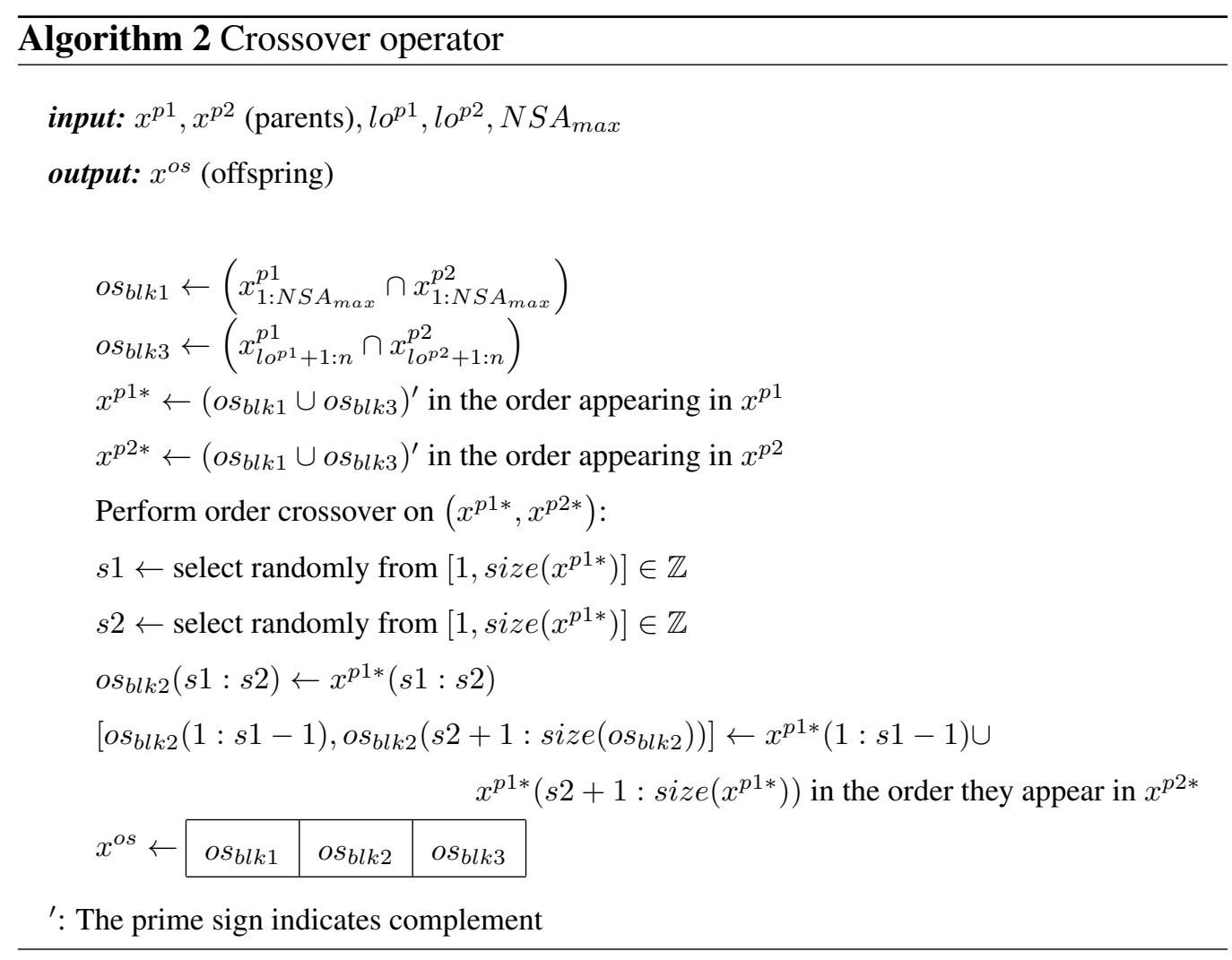




\section{Resilience model and analysis}

The application of $A M I G O$ for the optimization of disaster resilience of highway bridges is discussed in the following. First, a brief review of the current approaches for resilience analysis of bridge networks is presented. The new connectivity-based resilience metric and restoration model proposed for this study are described afterward.

\subsection{Previous models of highway network resilience}

The impact of damaged bridges on the performance of transportation networks can be quantified through traffic analysis on the damaged network, which is carried out by solving the well-known traffic distribution and assignment problems [38]. To this purpose, transportation networks are modeled in the form of directed graphs, in which nodes represent population centers (e.g., cities, business or residential centers) or road intersections and edges represent the connecting road segments. Solving the traffic distribution and assignment problem is an iterative procedure through which two fundamental sets of information about the traffic characteristics of the network are computed. In particular, traffic distribution uses a gravitational model [39] to estimate the number of trips between each pair of nodes, and traffic assignment distributes the generated and attracted trips to the roads by means of Wardrop's user equilibrium model [40], which provides the

traffic flow $\left(f_{i j}\right)$ and travel time $\left(c_{i j}\right)$ for each road segment in the network. More details about the computational algorithm used herein for traffic analysis are presented in [41, 42].

In the case of an extreme event, the damage induced by the disaster, safety considerations, or restoration activities might require to partially or fully close some 

the traffic characteristics of the network is modeled as an increase in the travel time of the road segments whose bridges are damaged, as shown in the following [28]:

$$
c_{i j}=c_{i j}^{0} \cdot\left[1+\alpha\left(\frac{f_{i j}}{f_{i j}^{c}}\right)^{\beta}\right]+\sum_{b \in i j}\left(1-Q_{b, i j}\right) \cdot c_{b}^{d} \cdot\left[1+\alpha\left(\frac{\left(1-Q_{b, i j}\right) \cdot f_{i j}}{f_{b}^{d}}\right)^{\beta}\right]
$$

The first part of Eq. (2) is actually the BPR latency function [43] that accounts for the time required to cover a highway segment in regular conditions (i.e. when all bridges of the segment are fully in service). In this part, $f_{i j}$ and $c_{i j}$ are the traffic flow and the time needed to cover segment $i j$, respectively. $c_{i j}^{0}$ is the time to cover the segment at free flow and $f_{i j}^{c}$ is the practical flow capacity of the segment. $\alpha$ and $\beta$ are model parameters set equal to 0.15 and 4.0, respectively [43]. The second part (summation) takes into account the additional time required by the traffic to bypass the damaged bridges. Parameters $f_{b}^{d}$ and $c_{b}^{d}$ are the practical capacity and the minimum time required to cover the detour of bridge $b$, respectively. $Q_{b, i j}$ is the functionality of bridge $b$, typically represented by the fraction of the total number of lanes of segment $i j$ which is open to traffic. For instance, the value of $Q_{b, i j}$ is 0 when a bridge is closed (out of service), 0.5 when half of the lanes are open (partially in service), and 1.0 when the bridge is fully open to the traffic (fully in service).

As a development of the concept of resilience triangle introduced in [14], the following equation has been proposed by Reed et al. [44] for quantifying the

$$
R=\frac{\int_{t_{0}}^{t_{0}+T} Q(t) d t}{T}
$$


where $Q(t)$ is the time-variant functionality of the system, $t_{0}$ is the time of occurrence of the extreme event, and $T$ is the investigated time span.

For networks of highway bridges, Bocchini and Frangopol [28] proposed the following functionality indicator, which measures the normalized performance difference between the post- and pre-event network:

$$
Q_{f l o w}(t)=\frac{\Gamma(t)-\Gamma^{0}}{\Gamma^{100}-\Gamma^{0}}
$$

where $\Gamma(t)$ is the network performance index at time $t . \Gamma^{0}$ and $\Gamma^{100}$ are the performance indices corresponding to the cases where all the bridges are in service $\left(Q_{b, i j}=1 \forall b\right)$, or out of service $\left(Q_{b, i j}=0 \forall b\right)$, respectively. The performance index $\Gamma(t)$ at each time instance is computed as follows:

$$
\Gamma(t)=\frac{1}{\gamma_{T} \cdot T T T(t)+\gamma_{D} \cdot T T D(t)}
$$

in which TTT and TTD are the total travel time spent and distance covered by all passengers traveling in the network in one hour, respectively. $\gamma_{T}$ and $\gamma_{D}$ are cost balancing factors associated with the time spent and the distance traveled by the passengers, measured in time ${ }^{-1}$ and distance ${ }^{-1}$, respectively. The values of TTT and TTD are computed using the following equations along with the procedure discussed earlier in this section:

$$
\begin{gathered}
T T T(t)=\sum_{i \in I} \sum_{j \in J} f_{i j}(t) \cdot c_{i j}\left[f_{i j}(t)\right] \\
T T D(t)=\sum_{i \in I} \sum_{j \in J}\left[f_{i j}(t) \cdot d_{i j}+\sum_{b \in i j} f_{i j}(t) \cdot\left(1-Q_{b, i j}\right) \cdot d_{b}^{d}\right]
\end{gathered}
$$

where $I$ is the set of all nodes of the network, and $J$ is the subset of nodes connected to node $i . d_{i j}$ is the length of the highway segment $i j$, and $d_{b}^{d}$ is the detour 
length of bridge $b$.

In real cases, disaster response activities are categorized into different phases based on their priority, as typically evaluated by decision makers and disaster managers [45, 46]. These phases, namely short-, medium-, and long-term are schematically shown in Figure 5. During the first phase, most of the efforts are focused on emergency and life saving activities, such as sheltering, evacuation, and hospitalization. Therefore, due to high criticality of the situation and short duration, no major restoration is feasible during the first phase and most of the restoration activities are planned for the following two phases. While the full restoration is the ultimate goal during the last stage, a minimum acceptable restoration of critical infrastructures is the main objective of the mid-term response phase. Accordingly, it is necessary to use appropriate functionality and resilience metrics in order to assess the quality of the response of the system with respect to the priorities of each restoration phase.

For the purpose of having a fair comparison of the network performance, as done by Eq. (4), the pre-event traffic demand is applied at all time instants, even though it is well known that the post-event demand is different. Therefore, this resilience index tends to reflect the quality of the response and restoration with respect to the last phase (long-term) of disaster management, whose ultimate goal is the complete restoration to the pre-event conditions.

\subsection{Proposed connectivity-based medium-term resilience}

The demand and behavior of traffic is evaluated by means of surveys or analytical models, such as the technique presented in Section 3.1. However, typically there is limited data available on the post-event travel demand. Also, the behavior of traffic in such situations is extremely complex as it is influenced by several 
socio-economic factors as well as evacuation and emergency policies. Therefore, the majority of analytical models fail to accurately capture post-disaster traffic characteristics. In particular, the models focusing on assessing the congestion by relying on origin-destination demands are not appropriate for short- or mediumterm emergency response evaluations [47, 48]. In this respect, in alternative to Eqs. (3) and (4), several other indices have been used in the literature to evaluate and compare the performance and resilience of transportation systems during emergency and recovery phases, among which connectivity- and accessibilitybased metrics are probably the most popular [47, 49,-52].

Typically, in each region there are certain locations that are of great importance due to social, economic, or topographic reasons. Emergency shelters, hospitals, airports, schools, and retail services are among these locations. Thus, in addition to their restoration, facilitating the traffic and enhancing the accessibility of these locations is always among the top priorities of disaster managers (after dealing with emergency situations in the first phase of the response). However, since in many cases these locations are not the major origin or destination of traffic in normal conditions, the restoration prioritization on the basis of maximizing the long-term resilience (computed using $Q_{\text {flow }}$ ) is not representative of the secondphase priorities.

The metric introduced in this paper is a time-variant connectivity-based measure $Q_{\text {conn }}(t)$ computed by the following equation:

$$
Q_{\text {conn }}(t)=\sum_{p=1}^{n p} C_{p} L_{p}(t)
$$

where $p$ is a node pair whose highway connectivity is assumed to have priority. $n p$ is the number of node pairs which need to be connected urgently. $C_{p}$ is a weight- 
ing factor reflecting the importance of fixing the connectivity between the nodes associated with pair $p$, presented in the form of percent of the total functionality $\left(Q_{\text {conn }}\right)$ that is achieved by restoring their connectivity. Therefore, assuming that fully connecting all selected pairs provides $100 \%$ functionality, the following constraint needs to be satisfied regarding $C_{p}$ :

$$
\sum_{p=1}^{n p} C_{p}=1
$$

The value of $C_{p}$ is determined based on engineering judgment; an example will be provided in Section 4. It is worth noting that the functionality computed using Eqs. (8) and (9) is associated with the level of connectivity among the specified urgent node pairs. In this context, full functionality does not necessarily reflect the case in which all the nodes of the network are connected, but only those important for the mid-term recovery. Parameter $L_{p}(t)$ determines the level of the connectivity between node pairs. In this respect, node pairs are considered fully or partially connected through network of highways if at least one route exists whose bridges are all fully (i.e. $Q_{b, i j}=1.0$ ) or at least partially (i.e. $Q_{b, i j}=0.5$ ) in service, respectively ("bottle neck assumption"). Accordingly, $L_{p}(t)$ is defined and computed at each time step as:

$$
L_{p}(t)= \begin{cases}1 & \text { if nodes of pair } p \text { are fully connected } \\ 0.5 & \text { if nodes of pair } p \text { are partially connected } \\ 0 & \text { if nodes of pair } p \text { are not connected }\end{cases}
$$

While the values of $L_{p}(t)$ in Eq. (10) have been set as consistent with the bridge functionalities $\left(Q_{b, i j} \in\{0.0,0.5,1\}\right)$, they can be adjusted for any particular application, based on the utilized bridge functionality model and connectivity consid- 
erations. At the end, the resilience computed using Eqs. (3) and (8, 10) addresses the medium-term restoration needs.

\subsection{Proposed bridge restoration model}

The restoration model provides the time required to restore a bridge to a certain level of functionality. In this study, the bridge restoration model is assumed to follow a stepwise function, which can be constructed by obtaining the time needed to partially $t\left(Q_{b, i j}=0.5\right)$ and fully $t\left(Q_{b, i j}=1.0\right)$ restore a bridge, as well as the replacement cost of the bridge. In general, more intermediate steps can be added, if deemed appropriate for a particular case. In order to obtain these points, a technique similar to the methodology presented in HAZUS-MH [53] has been used. Different restoration functions have been generated considering the functionality of bridges with respect to the carried and crossed traffic. To this purpose, it has been assumed that for bridges that carry the traffic, the desired points lie on normal CDF curves fitted to the discrete mean restoration time values provided by ATC-13 [54]. Therefore, the curve fitting has been carried out for four levels of initial damage states (i.e. slight, moderate, extensive, and complete damage), as well as two classes of bridges, namely SC-25a and SC-25c, associated with major and conventional bridges, respectively. Figure 6 illustrates the restoration data from ATC-13 and fitted curves for the class SC-25c.

375 Typically, for traffic carrying bridges, after a 50\% restoration of the functionality, the major required activities on the substructure are completed and the remaining operations are performed on the superstructure of the bridge. At this point, while half of the lanes are considered to be closed to the carried traffic, it can be assumed that the construction activities would not interfere with the crossed traffic. Therefore, the bridge restoration functions generated considering 
the functionality with respect to the carried traffic have been modified accordingly, to obtain the associated functionality with respect to the crossed traffic. Additionally, the time required to restore $50 \%$ of the carried traffic capacity for moderately damaged bridges is typically very short (e.g., less than a month). Therefore, it has been assumed that moderately damaged bridges are 50\% and $100 \%$ functional immediately after the extreme event, for traffic carried and crossed, respectively. Moreover, since the restoration of initially slightly damaged bridges does not require major construction activities, the restoration of these bridges has been ignored in this study, by assuming full functionality. Table 1 presents the key points of the restoration functions generated as discussed above for conventional bridges.

Based on the way data were collected by ATC, it has been assumed that the resulting stepwise curves are representative of the restoration functions of a bridge whose cost is equal to the mean replacement cost of all bridges in the studied region (e.g., California in this study). Therefore, assuming that the restoration time and effort are proportional to the cost of the bridges, an additional scaling factor has been applied to the resulting restoration times based on the replacement cost for each individual bridge:

$$
t_{c l, r c_{b}}^{D S^{0}}\left(Q_{b, i j}\right)=\frac{r c_{b}}{\overline{r c}_{c l}} \cdot t_{c l, \overline{r c}_{c l}}^{D S^{0}}\left(Q_{b, i j}\right)
$$

where $t_{c l, r c_{b}}^{D S^{0}}\left(Q_{b, i j}\right)$ is the time required to restore functionality $Q_{b, i j}$ of bridge $b$ of class $c l$ (major or conventional) with initial damage state $D S^{0}$ and restoration cost of $r c_{b} . \overline{r c}_{c l}$ is the mean replacement cost of class $c l$ bridges in the region.

\section{Numerical example}

The application of $A M I G O$ and the enhanced resilience model is illustrated in this section through a numerical example. The problem statement, input data, and 
the results of the resilience optimization are presented in the following. the practical capacity $f_{i j}^{c}$ of each link has been set to $2000 \frac{\text { car-equivalent }}{\text { vehicle.hour.lane }}$. A detailed discussion about the determination of other traffic parameters can be found 
in [41].

The network shown in the figure contains 238 highway bridges. The detour distance $d_{b}^{d}$, and replacement cost $r c_{b}$ of each bridge have been extracted from the National Bridge Inventory [56]. Based on the characteristics of the surrounding secondary roads, the practical capacity of the detours have been set to 1500 $\frac{\text { car-equivalent }}{\text { vehicle.hour.lane }}$.

A magnitude 7 earthquake scenario has been simulated using HAZUS-MH [53]. The epicenter of the event has been assumed to be located at latitude $32.78^{\circ}$ and longitude $-117.15^{\circ}$, as shown schematically in Figure 7 . A fragility analysis providing the probability of exceeding each damage level $(D S)$ has been carried out using HAZUS-MH. In order to conservatively determine a representative damage level $(D S)$ based on the results of the fragility analysis, the following assumptions have been made:

$$
D S^{0}= \begin{cases}\text { complete } & \text { if } \operatorname{Probability}(D S=\text { comp. }) \geq 0.15 \\ \text { extensive } & \text { else if Probability }(D S \geq \text { ext. }) \geq 0.25 \\ \text { moderate } & \text { else if Probability }(D S \geq \text { mod. }) \geq 0.30 \\ \text { slight } & \text { else if Probability }(D S \geq \text { sl. }) \geq 0.40 \\ \text { no damage } & \text { otherwise }\end{cases}
$$

As already mentioned, slight damage does not affect the functionality of the bridges. Hence, the optimization algorithm has to consider only the 80 bridges with at least moderate damage. Table 2 presents the properties of these bridges, along with their associated ID (as specified by HAZUS-MH) which can easily be used to track the location and characteristics of each bridge in the network.

It has been assumed that node 1 shown in Figure 7 represents the port and, 
therefore, all business trips associated with the port are originated from or attracted to this node. Also, nodes 9, 15, and 16 act as the gates from which the consignments can be shipped to their major destinations. Thus, it is of great importance to restore connectivity between the gates and the port as soon as possible, in order to facilitate the shipping process from and to the port. Therefore, in addition to the long-term resilience of the transportation network $R\left[Q_{\text {flow }}\right]$ computed using Eqs. (3) and (4), maximizing the resilience associated with connecting the port node and the gate nodes $R\left[Q_{\text {conn }}\right]$ (medium-term resilience), computed using Eqs. (3) and (8), has been considered as the objective of this example. Restoration of the connectivity between the port and each gate has been assumed to have the same importance (i.e. $C_{p}=0.167, p \in\{1-9,9-1,1-15,15-1,1-$ $16,16-1\})$. In terms of constraints, it has been assumed that 5 major bridge construction companies are available $\left(N S A_{\max }=5\right)$ to restore the damages in 3 years ( $T=36$ months). Resilience computations have been performed with the time step of one month.

\subsection{Multi-objective resilience optimization results}

The greedy algorithm discussed earlier has been run to determine a convenient initial population. The first level of search has been carried out considering only the restoration of 2 bridges $\left(l_{\text {greedy }}^{0}\right)$. For subsequent steps, the partial solutions have been generated by adding one bridge (i.e. $\Delta l_{\text {greedy }}=1$ ) to the optimal partial solutions obtained from the previous search level. Figure 8 shows the values of the two objectives for the examined non-optimal partial solutions along with the Pareto-front of each level. The outcome of the algorithm consists in two restoration schedules with the same values of objectives equal to $(40.56 \%, 50.0 \%)$ and $l o^{s}=47$ (Figure 8). 
The individuals obtained from the greedy algorithm have been added to 998 random individuals, for a total of 1000 individuals for the first generation. AMIGO converged after 100 generations to a set of Pareto optimal solutions. At each generation, $A M I G O$ allows to find a set of trial solutions that have higher objective values compared to those evaluated previously. Figure 9 presents the Pareto front as well as the non-optimal solutions evaluated through the process. As shown in Figure 9b there is little difference between the values of objectives at different points in the Pareto front. However, even a tiny difference causes considerable impact on the performance and socio-economic losses after an event. These solutions allow different trade-offs between the medium-term (phase-2) and long-term (phase-3) restoration priorities. The Pareto front contains 11 distinct points, but the total number of optimal restoration plans is 128 , since for most of the points in the Pareto front more than one trial solution (restoration plan) have been provided by the algorithm. This allows decision makers to select the restoration plan that meets the resilience criteria, while taking into account also other factors (e.g., socio-economic or environmental) which are not considered in the formulation of the problem. As an example, one representative restoration plan has been selected and will be discussed in detail. Solution $s^{*}$, shown in Figure 9p with associated objective values of $(44.80 \%, 77.55 \%)$, has been chosen for this purpose. In total, 7 restoration plans have been provided for this point (with the same objective values). Figure 10 shows one of the bridge restoration sequences associated with this solution. According to this solution, the restoration of 60 (out of 80) bridges is feasible $\left(l o^{s}=60\right)$, while the restoration of the rest of the bridges placed in block-3 is either not initiated, or not completed to the level which improves the functionality of the bridge. 
In order to evaluate the work flow related to this restoration plan, the associated Gantt chart is illustrated in Figure 11. This figure shows the restoration initiation times and the duration of partial and full restorations for the bridges of block-1 and block-2 of the sequence shown in Figure 10 . The restoration starts simultaneously for all bridges of block-1 $(21,12,19,8,58)$ and continues whenever a construction company becomes available, considering that $N S A_{\max }=5$. Regardless of the numerical value, that changes for different situations, this constraint provides more realistic results and addresses the issue of the previous technique presented in [29] in which most of the restorations started immediately after the event (as pointed out in [57, 58]). The figure shows that the partial restoration of bridge 9 has been completed by the end of month 26 after the initiation of the network restorations. However, completion of the restoration for this bridge is not achievable before the time span. On the other hand, after the full restorations of bridges $51,46,47$, and 54 have been completed, there is no bridge whose restoration can be started and at least partially completed. Thus, since any restoration at this point will not have impact on the resilience indices of the network, the restoration schedule of the rest of the bridges have not been illustrated in the figure. However, it is expected that the restoration activities would continue even after the 36 months $(T)$.

Figure 12 shows the evolution of the two functionality measures $Q_{\text {flow }}(t)$ and $Q_{\text {conn }}(t)$ for solution $s^{*}$, associated with the two resilience objectives. Figure 12 illustrates that the long-term functionality of the network has not been revived to 520 its pre-event condition (i.e. 100\%), since the full restoration of all bridges is not possible during 36 months with the available resources. As shown in Figure 12b, the highway connection of the critical points of the region (here the port and the 
gate nodes) has been completely lost immediately after the extreme event (i.e. $\left.Q_{\text {conn }}\left(t_{0}\right)=0\right)$. However, by implementing the restoration plan $s^{*}$, it is possible to fully connect these points through highways in 12 months. The major increases in both objectives $Q_{\text {flow }}$ and $Q_{\text {conn }}$ are observed in months 6 and 10 after the restoration process starts.

\subsection{Convergence analysis and comparison}

It is useful to evaluate the performance of evolutionary algorithms by studying the optimality of the final solution, as well as the quality of the convergence to the final solution. In the case of the current example, AMIGO converged after evaluating about 100,000 trial solutions, which is a tiny fraction $(1.4 e-112)$ of all possible sequences (equal to 80 !). Also, by observing the distribution of the examined solutions in the design space shown in Figure $9 \mathrm{a}$, it can be seen that $A M I G O$ has concentrated most of the effort to search the solutions in the neighborhood of the Pareto front. The General Distance (GD) measure has been utilized to assess the performance of the developed algorithm and search progress in a quantitative manner. GD is a quality indicator for Multi-Objective Evolutionary Algorithms (MOEA) which quantifies the average minimum distance between a set of Pareto optimal solutions $\left(P F_{\text {known }}\right)$ obtained by the evolutionary algorithm, and the global Pareto optimal solutions $\left(P F_{\text {true }}\right)$ known a priori [32], as follows:

$$
G D=\frac{\sqrt{\left(\sum_{i=1}^{n} d_{i}^{2}\right)}}{\left|P F_{\text {known }}\right|}
$$

where $d_{i}$ is the shortest Euclidean distance between the member $i$ from $P F_{\text {known }}$, and members of $P F_{\text {true }} .\left|P F_{\text {known }}\right|$ is the number of the solutions in $P F_{\text {known }}$. For the case of the current problem, the above indicator has been computed for the

Pareto fronts obtained at the end of each generation $\left(P F_{\text {current }}\right)$, assuming that the 
Pareto front to which $A M I G O$ has converged (Figure 9) is $P F_{\text {true }}$. In this way, it is possible to monitor the evolution of the objectives through the generations and evaluate the performance of the algorithm for multi-objective problems, although there is no guarantee that the obtained Pareto front is the actual $P F_{\text {true }}$. Figure 13 shows that the value of GD is large at the beginning, but it decreases quickly during the first 25 generations. This indicates that while the search has started from a region relatively far from the optimal solution, the algorithm managed to approach the neighborhood of the Pareto front shown in Figure 9 $\mathrm{b}$ in a limited number of trials. Although the algorithm continuously provides improved solutions at each generation, the value of GD does not show considerable improvements after generation 25. Therefore, in case the amount of available computational capacity is limited, the algorithm can be terminated earlier, when the provided solution is already in close proximity of the Pareto optimal front found after 100 generations. Moreover, the optimality of the solution provided by $A M I G O$ has been validated with results given by exhaustive search for simpler problems. For all the investigated cases, $A M I G O$ was able to converge to the true Pareto front. For the Port of San Diego the total number of possible bridge sequences (including redundant solutions) is too large $(80 !>7 e 118)$ to find the Pareto front by exhaustive search.

The overall performance of $A M I G O$ has been also compared to two other formulations presented in [31, 57]. Bocchini [57] formulated the problem in the $\mathbb{R}^{n b}$ domain, by introducing the bridge restoration priority $0 \leq \rho_{b} \leq 1$ as the design variable of the problem. Karamlou and Bocchini [31] used a combinatorial formulation and genetic algorithms with classic swap and order crossovers [37]. In the following, these two methodologies are called B2013 and KB2014, respectively. It is worth noting that both KB2014, and B2013 are based on a standard imple- 
mentation of Genetic Algorithms [59], meaning that they only use the value of the objective function(s) as a criterion for reproduction and searching the design domain, whereas $A M I G O$ adds multiple-input generic operators. Resilience optimization analyses have been carried out on San Diego port transportation network as discussed in this section, considering 1000 populations and 100 generations. To have a clearer comparison and allow the previous methodologies to converge for this large network, the problem has been reduced to single-objective optimization $\left(R_{\text {conn }}\right)$. Figure 14 shows the values of the best fitness after each generation for the three mentioned formulations. The best fitness provided by B2013, KB2014, and $A M I G O$ is $76.62 \%, 78.00 \%$, and $79.63 \%$ respectively. Therefore, the results show that in terms of optimality $A M I G O$ is superior compared to the other two techniques. It is worth mentioning that the optimum value obtained by $A M I G O$ for $R\left(Q_{\text {conn }}\right)$ is consistent with the maximum value of this objective computed for the multi-objective optimization problem shown in Figure 9p. Additionally, $A M I G O$ shows improvement in the convergence rate. The figure shows that the value of the objective decreases continuously and quickly in $A M I G O$ and stabilizes in about 10 generations. The overall pace of reduction in B2013 and KB2014 is substantially slower.

\section{Conclusion}

In this paper, the formulation of a scheduling problem considering resource and time constraints is presented in detail and a novel evolutionary solution methodology is proposed. The proposed optimization algorithm is called Algorithm with Multi-Input Genetic Operators (AMIGO) as it takes advantage of the additional data in the genetic operators to make the search process more efficient and expe- 
dite the convergence. Several developed optimization operators for $A M I G O$, such as initial population generator, mutation and crossover, are presented for the first time in this manuscript. The proposed methodology can be used for solving the optimization problems with any type and number of objective functions.

The application of the methodology to resilience optimal post-disaster restoration intervention of damaged highway bridges is presented. Through this application, a number of enhancements have been made to the framework and formulation of resilience analysis of highway networks. In addition to the long-term resilience metric previously used for resilience evaluation of bridge networks, a connectivity-based resilience indicator is introduced, to take into account the restoration priorities typically considered during the medium-phase of disaster management activities. Moreover, a new bridge recovery model is proposed. Compared to the previous studies, this recovery model is more realistic, as it takes advantage of the available restoration functions obtained by experts surveys and scaling factors that account for the bridge cost. The San Diego port transportation network was chosen for the demonstration of the performance of the proposed methodology. The number of damaged bridges in this example is considerably higher compared to the previous works involving restoration optimization with traffic analysis.

While the total number of feasible solutions for the examined problem is considerably large (80!), the result of a convergence analysis shows that the algorithm managed to find a set of near-optimal Pareto solutions in a small number of trials (about 25 generation). A comparison is made between the performance of the $A M I G O$ optimization formulation and previous works presented in [31, 57]. The results testify the improvement made by the current optimization approach, 
both in terms of optimality of the solution and convergence rate. This is of great importance, since for large realistic networks, the traffic analysis procedure can be computationally very expensive. Therefore, reducing the number of required generations for convergence can considerably affect the computational cost of the problem and make this approach finally applicable to real-size networks. Compared to previous formulations [28, 29], the use of operational resource constraints and the new recovery model yield the generation of more realistic schedules.

Several other improvements can expand and enhance the optimization approach and resilience models proposed in this study. These include consideration of task precedence constraints (if any), as well as gap between the operations in the schedule due to mobilization of crews and equipment. Also, the effect of uncertainty in the characteristics of the activities should be investigated. For instance, in case of disaster resilience evaluation, a fully probabilistic resilience analysis is required to take into account the uncertainty in hazard, damage level, and restoration of bridges.

\section{Acknowledgments}

The support provided by the National Science Foundation through award CMMI - 1541177, the P.C. Rossin College of Engineering and Applied Science through the Rossin Doctoral Fellowship, the Department of Civil and Environmental Engineering, and the ATLSS Engineering Research Center at Lehigh University is gratefully acknowledged.

The opinions and conclusions presented in this paper are those of the authors and do not necessarily reflect the views of the sponsoring institutions. 


\section{References}

[1] K. R. Baker, Introduction to sequencing and scheduling, John Wiley \& Sons, 1974.

[2] E. Demeulemeester, W. Herroelen, Project scheduling: A research handbook, Kluwer Academic Publishers, 2002.

[3] R. Klein, Scheduling of Resource-Constrained Projects, Operations Research/Computer Science Interfaces Series, Kluwer Academic Pubslishers, 2000.

[4] K. Neumann, C. Schwindt, J. Zimmermann, Project scheduling with time windows and scarce resources: temporal and resource-constrained project scheduling with regular and nonregular objective functions, Springer Berlin, 2003.

[5] R. Kolisch, R. Padman, An integrated survey of deterministic project scheduling, Omega 29 (3) (2001) 249-272.

[6] S. Hartmann, D. Briskorn, A survey of variants and extensions of the resource-constrained project scheduling problem, European Journal of Operational Research 207 (1) (2010) 1-14.

[7] L. Özdamar, G. Ulusoy, A survey on the resource-constrained project scheduling problem, IIE transactions 27 (5) (1995) 574-586.

[8] P. Brucker, A. Drexl, R. Möhring, K. Neumann, E. Pesch, Resourceconstrained project scheduling: Notation, classification, models, and methods, European journal of operational research 112 (1) (1999) 3-41. 
[9] C. D. Poland, Building Resilient Communities: Fresh Challenges for Earthquake Professionals, Lawson Lecture Series, UC Berkeley, Berkeley, CA, USA, April 14, 2009, complete video available at: http:// seismo.berkeley.edu/news/lawsonlecture.html, 2009.

[10] J. Kim, S. S. Oh, The virtuous circle in disaster recovery: who returns and stays in town after disaster evacuation?, Journal of Risk Research 17 (2014) $665-682$.

[11] C. S. Holling, Resilience and stability of ecological systems, Annual Review of Ecology and Systematics 4 (1973) 1-23.

[12] D. Ludwig, D. D. Jones, C. S. Holling, Qualitative analysis of insect outbreak systems: The spruce budworm and forest, Journal of Animal Ecology 47 (1) (1978) 315-332.

[13] Presidential Policy Directive, Critical Infrastructure Security and Resilience. PPD-21, Released February 12, 2013, available at: http://www.whitehouse.gov/the-press-office/2013/ 02/12/presidential-policy-directive-criticalinfrastructure-security-and-resil Accessed Oct 20, 2014, 2013.

[14] M. Bruneau, S. E. Chang, R. T. Eguchi, G. C. Lee, T. D. ORourke, A. M. Reinhorn, M. Shinozuka, K. Tierney, W. A. Wallace, D. von Winterfeldt, A framework to quantitatively assess and enhance the seismic resilience of communities, Earthquake Spectra 19 (4) (2003) 733-752. 
[15] S. E. Chang, M. Shinozuka, Measuring improvements in the disaster resilience of communities, Earthquake Spectra 20 (2004) 739-755.

[16] M. Ouyang, L. Dueñas-Osorio, X. Min, A three-stage resilience analysis framework for urban infrastructure systems, Structural Safety 3637 (2012) 23-31.

[17] C. W. Zobel, Representing perceived tradeoffs in defining disaster resilience, Decision Support Systems 50 (2011) 394-403.

[18] D. Henry, J. Emmanuel Ramirez-Marquez, Generic metrics and quantitative approaches for system resilience as a function of time, Reliability Engineering \& System Safety 99 (2012) 114-122.

[19] R. Francis, B. Bekera, A metric and frameworks for resilience analysis of engineered and infrastructure systems, Reliability Engineering \& System Safety 121 (2014) 90-103.

[20] R. Faturechi, E. Miller-Hooks, A mathematical framework for quantifying and optimizing protective actions for civil infrastructure systems, ComputerAided Civil and Infrastructure Engineering 29 (8) (2014) 572-589.

[21] M. Cavallaro, D. Asprone, V. Latora, G. Manfredi, V. Nicosia, Assessment of urban ecosystem resilience through hybrid socialphysical complex networks, Computer-Aided Civil and Infrastructure Engineering 29 (8) (2014) 608625 .

[22] P. Franchin, F. Cavalieri, Probabilistic assessment of civil infrastructure resilience to earthquakes, Computer-Aided Civil and Infrastructure Engineeringdoi: $10.1111 / \mathrm{mice} .12092$. 
[29] P. Bocchini, D. M. Frangopol, Restoration of bridge networks after an earthquake: Multicriteria intervention optimization, Earthquake Spectra 28 (2) (2012) 426-455. 
[30] A. Venkittaraman, S. Banerjee, Enhancing resilience of highway bridges through seismic retrofit, Earthquake Engineering \& Structural Dynamics 43 (8) (2014) 1173-1191.

[37] M. Gen, R. Cheng, Genetic algorithms and engineering optimization, John Wiley \& Sons, New York, 2000.

[31] A. Karamlou, P. Bocchini, Optimal bridge restoration sequence for resilient transportation networks, in: Structures Congress 2014, American Society of Civil Engineers, 2014, pp. 1437-1447.

[32] C. A. C. Coello, D. A. Van Veldhuizen, G. B. Lamont, Evolutionary algorithms for solving multi-objective problems, Vol. 242, Springer, 2002.

[33] The Mathworks Inc., Matlab version 8.3 - R2014a, Natick, Massachusetts, 2014.

[34] K. Deb, T. Goel, Controlled elitist non-dominated sorting genetic algorithms for better convergence, in: E. Zitzler, L. Thiele, K. Deb, C. A. C. Coello, D. Corne (Eds.), Evolutionary Multi-Criterion Optimization, Lecture Notes in Computer Science, Springer Berlin Heidelberg, 2001, pp. 67-81.

[35] K. Deb, A. Pratap, S. Agarwal, T. Meyarivan, A fast and elitist multiobjective genetic algorithm: NSGA-II, IEEE transactions on evolutionary computation 6 (2) (2002) 182197.

[36] D. F. Rothlauf, Representations for genetic and evolutionary algorithms, in: Representations for Genetic and Evolutionary Algorithms, Springer Berlin Heidelberg, 2006. 
[38] S. P. Evans, Derivation and analysis of some models for combining trip distribution and assignment, Transportation Research 10 (1) (1976) 37-57.

[39] D. M. Levinson, A. Kumar, Multimodal trip distribution: structure and application, Transportation Research Record 1466 (1994) 124-124.

[40] Y. Sheffi, Urban Transportation Networks: Equilibrium Analysis With Mathematical Programming Methods, Prentice Hall, 1985.

[41] P. Bocchini, D. M. Frangopol, A stochastic computational framework for the joint transportation network fragility analysis and traffic flow distribution under extreme events, Probabilistic Engineering Mechanics 26 (2) (2011) $182-193$.

[42] D. Saydam, P. Bocchini, D. M. Frangopol, Time-dependent risk associated with deterioration of highway bridge networks, Engineering Structures 54 (2013) 221-233.

[43] Bureau of Public Roads, Traffic Assignment Manual, U.S. Department of Commerce, Urban Planning Division, Washington D.C., 1964.

[44] D. Reed, K. Kapur, R. Christie, Methodology for assessing the resilience of networked infrastructure, IEEE Systems Journal 3 (2) (2009) 174-180.

[45] C. D. Poland, The resilient city: defining what San Francisco needs from its seismic mitigation policies, San Francisco Planning and Urban Research Association report, San Francisco, CA, USA, 2009.

[46] DHS Risk Steering Committee, DHS risk Lexicon, Washington, DC: The Department of Homeland Security, 2008. 
[47] S. E. Chang, N. Nojima, Measuring post-disaster transportation system performance: the 1995 kobe earthquake in comparative perspective, Transportation Research Part A: Policy and Practice 35 (6) (2001) 475-494.

[48] L. Chang, A. S. Elnashai, B. F. Spencer, Post-earthquake modelling of transportation networks, Structure and Infrastructure Engineering 8 (10) (2012) 893-911.

[49] S. Giovinazzi, A. Nicholson, Transport network reliability in seismic risk analysis and management., Tech. rep., University of Canterbury. Civil and Natural Resources Engineering (2010).

[50] J. Sohn, Evaluating the significance of highway network links under the flood damage: An accessibility approach, Transportation Research Part A: Policy and Practice 40 (2006) 491-506.

[51] D. Tuzun Aksu, L. Ozdamar, A mathematical model for post-disaster road restoration: Enabling accessibility and evacuation, Transportation Research Part E: Logistics and Transportation Review 61 (2014) 56-67.

[52] P. Bocchini, D. M. Frangopol, Connectivity-based optimal scheduling for maintenance of bridge networks, Journal of Engineering Mechanics 139 (6) (2013) 760-769.

[53] DHS, HAZUS-MH MR4 Earthquake Model User Manual, Department of Homeland Security; Federal Emergency Management Agency; Mitigation Division. Whashington, D.C. (2009).

[54] ATC, Earthquake damage evaluation data for California, Tech. rep., Applied Technology Council, ATC-13, Redwood City, CA (1985). 
[55] AAPA, U.S. Port Waterborne Foreign Trade, American Association of Port Authorities, available at: http://www.aapa-ports.org/ Industry/content.cfm? It emNumber=900, 2013.

[56] FHWA, National Bridge Inventory, Federal HighWay Administration, available on line at http://www.fhwa.dot.gov/bridge/nbi.htm (visited on June 15, 2014), 2013.

[57] P. Bocchini, Computational procedure for the assisted resilience-oriented disaster management of transportation systems, in: G. Deodatis, B. R. Ellingwood, D. M. Frangopol (Eds.), Safety, Reliability, Risk and Life-Cycle Performance of Structures and Infrastructures, CRC Press, Taylor and Francis Group, Colombia University, NY, 2013.

[58] E. D. Vugrin, M. A. Turnquist, N. J. Brown, Optimal recovery sequencing for enhanced resilience and service restoration in transportation networks, Int J of Critical Infrastructures.

[59] D. E. Goldberg, Genetic Algorithms in Search, Optimization, and Machine Learning, Addison-Wesley Professional, 1989. 
Table 1: Restoration function values for mean cost bridges

\begin{tabular}{ccccc}
\hline \hline \multirow{2}{*}{$D S^{0}$} & \multicolumn{2}{c}{$t\left[Q_{b}=0.5\right]_{\text {in days }}$} & \multicolumn{2}{c}{$t\left[Q_{b}=1.0\right]_{\text {in days }}$} \\
\cline { 2 - 5 } & $\begin{array}{c}\text { Carried } \\
\text { traffic }\end{array}$ & $\begin{array}{c}\text { Crossed } \\
\text { traffic }\end{array}$ & $\begin{array}{c}\text { Carried } \\
\text { traffic }\end{array}$ & $\begin{array}{c}\text { Crossed } \\
\text { traffic }\end{array}$ \\
\hline Complete & 126 & 126 & 304 & 126 \\
Extensive & 46 & 46 & 85 & 46 \\
Moderate & 0 & 0 & 9 & 0 \\
Slight & 0 & 0 & 0 & 0 \\
\hline \hline
\end{tabular}


Table 2: Selected damaged bridges and their properties

\begin{tabular}{|c|c|c|c|c|c|c|c|c|c|}
\hline No. & Bridge ID & $r c_{b}(\mathrm{M} \$)$ & $d_{b}^{d}(\mathrm{Km})$ & $D S_{b}^{0}$ & No. & Bridge ID & $r c_{b}(\mathrm{M} \$)$ & $d_{b}^{d}(\mathrm{Km})$ & $D S_{b}^{0}$ \\
\hline 1 & CA028824 & 2.634 & 3 & Extensive & 41 & CA028616 & 3.347 & 2 & Moderate \\
\hline 2 & $\mathrm{CA} 028460$ & 2.389 & 2 & Moderate & 42 & CA028619 & 3.425 & 2 & Moderate \\
\hline 3 & CA028416 & 1.789 & 2 & Moderate & 43 & CA028462 & 2.281 & 2 & Moderate \\
\hline 4 & CA028580 & 1.303 & 4 & Complete & 44 & CA028429 & 11.749 & 3 & Extensive \\
\hline 5 & CA028560 & 3.307 & 4 & Complete & 45 & CA029994 & 7.845 & 2 & Moderate \\
\hline 6 & CA028562 & 0.843 & 3 & Extensive & 46 & CA029429 & 9.543 & 2 & Moderate \\
\hline 7 & CA028665 & 1.071 & 4 & Complete & 47 & CA029433 & 9.404 & 2 & Moderate \\
\hline 8 & CA029857 & 4.522 & 3 & Extensive & 48 & CA029436 & 2.321 & 2 & Moderate \\
\hline 9 & CA029207 & 4.622 & 4 & Complete & 49 & CA029445 & 47.168 & 2 & Moderate \\
\hline 10 & CA028599 & 3.906 & 2 & Moderate & 50 & CA029454 & 7.384 & 2 & Moderate \\
\hline 11 & CA028601 & 6.276 & 2 & Moderate & 51 & CA029439 & 47.168 & 2 & Moderate \\
\hline 12 & CA028593 & 3.138 & 4 & Complete & 52 & CA029455 & 3.213 & 2 & Moderate \\
\hline 13 & CA028586 & 2.179 & 4 & Complete & 53 & CA029777 & 9.424 & 2 & Moderate \\
\hline 14 & CA028828 & 3.698 & 4 & Complete & 54 & CA029789 & 3.323 & 2 & Moderate \\
\hline 15 & CA028533 & 11.257 & 4 & Complete & 55 & CA028659 & 4.649 & 4 & Complete \\
\hline 16 & CA028531 & 5.797 & 2 & Moderate & 56 & CA029780 & 1.280 & 2 & Moderate \\
\hline 17 & CA028527 & 2.097 & 4 & Complete & 57 & CA028657 & 4.413 & 4 & Complete \\
\hline 18 & CA028591 & 5.330 & 4 & Complete & 58 & CA029791 & 1.126 & 3 & Extensive \\
\hline 19 & CA029343 & 6.890 & 3 & Extensive & 59 & CA029784 & 19.838 & 2 & Moderate \\
\hline 20 & CA029341 & 3.615 & 2 & Moderate & 60 & CA029351 & 8.220 & 2 & Moderate \\
\hline 21 & CA028921 & 28.214 & 2 & Moderate & 61 & CA029332 & 7.162 & 2 & Moderate \\
\hline 22 & CA029352 & 13.728 & 4 & Complete & 62 & CA029329 & 7.162 & 2 & Moderate \\
\hline 23 & CA028652 & 1.590 & 4 & Complete & 63 & CA029345 & 2.700 & 2 & Moderate \\
\hline 24 & CA028939 & 1.866 & 4 & Complete & 64 & CA028954 & 1.087 & 2 & Moderate \\
\hline 25 & CA029478 & 2.700 & 4 & Complete & 65 & CA028961 & 14.371 & 4 & Complete \\
\hline 26 & CA028948 & 6.434 & 2 & Moderate & 66 & CA028964 & 1.571 & 2 & Moderate \\
\hline 27 & CA028704 & 1.636 & 4 & Complete & 67 & CA028962 & 2.127 & 2 & Moderate \\
\hline 28 & CA028605 & 1.012 & 2 & Moderate & 68 & CA028965 & 1.223 & 2 & Moderate \\
\hline 29 & CA029308 & 3.953 & 2 & Moderate & 69 & CA028845 & 2.417 & 2 & Moderate \\
\hline 30 & CA029306 & 3.912 & 2 & Moderate & 70 & CA028955 & 1.641 & 2 & Moderate \\
\hline 31 & CA029312 & 1.957 & 2 & Moderate & 71 & CA029361 & 8.960 & 2 & Moderate \\
\hline 32 & CA029698 & 3.601 & 2 & Moderate & 72 & CA029861 & 0.979 & 2 & Moderate \\
\hline 33 & CA029305 & 7.145 & 2 & Moderate & 73 & CA029859 & 1.346 & 2 & Moderate \\
\hline 34 & CA029191 & 4.175 & 2 & Moderate & 74 & CA029358 & 8.876 & 2 & Moderate \\
\hline 35 & CA029199 & 3.195 & 2 & Moderate & 75 & CA029860 & 0.979 & 2 & Moderate \\
\hline 36 & CA028475 & 2.890 & 2 & Moderate & 76 & CA029865 & 10.087 & 3 & Extensive \\
\hline 37 & CA028617 & 5.770 & 4 & Complete & 77 & CA029939 & 10.200 & 2 & Moderate \\
\hline 38 & CA028428 & 26.183 & 3 & Extensive & 78 & CA028415 & 1.286 & 3 & Extensive \\
\hline 39 & CA028614 & 0.658 & 2 & Moderate & 79 & CA029339 & 3.125 & 2 & Moderate \\
\hline 40 & CA028612 & 5.063 & 2 & Moderate & 80 & CA029476 & 2.676 & 4 & Complete \\
\hline
\end{tabular}




\section{List of Figures}

1 Schematic view of $A M I G O$. The highlighted arrows indicate the novel elements of the framework. The use of additional informa-

\begin{tabular}{|c|} 
novel elements of the framework. The use of additional informa- \\
tion in the genetic operators enables a much faster convergence to
\end{tabular}

820 the optimal solution. . . . . . . . . . . . 42

.

2 Flowchart of $A M I G O$ for multi-objective optimization. The high-

lighted sections and arrows indicate the new aspects of the algo-

rithm. Note that the two selection processes shown in the figure are different and satisfy different purposes: the New Generation

825 \begin{tabular}{|l}
\hline \hline Selection is carried out to reduce the size of the mixed population \\
\hline \hline (equal to $2 \cdot$ popsize) to the original popsize, then the Reproduc-
\end{tabular} tion Selection is performed to choose parents used in reproduction functions (i.e. mutation and crossover). . . . . . . . . . . 43

$3 \quad$ Trial solution representation $\ldots \ldots . . \ldots 44$

$4 \quad$ Greedy algorithm optimization example for a 4-activity project

830

5 Disaster management phases . . . . . . . . . . . . . 46

6 Restoration model for carrying bridges: fitted normal CDF (gray curves), stepwise restoration curves used in this study (black lines). 47

$7 \quad$ Port of San Diego and the modeled transportation network . . . . 48

$835 \quad 8 \quad$ Greedy algorithm preliminary optimization $\ldots \ldots . . \ldots 49$

$9 \quad$ AMIGO optimization results: (a) Trial solutions, (b) Pareto-front . 50

Solution $s^{*}$ (numbers in the sequence are bridge numbers as shown

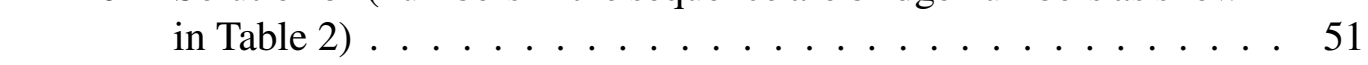

11 Gantt chart for solution $s^{*} \ldots \ldots$. . . . . . . . . 52

12 Evolution of the two functionality indicators for solution $s^{*}$ : (a) $Q_{\text {flow }}(t),(\mathrm{b}) Q_{\text {conn }}(t) \ldots \ldots \ldots \ldots . \ldots . \ldots 53$

13 Evolution of the GD indicator . . . . . . . . . . . 54

14 Best fitness values at each generation for three methodologies. . . 55 


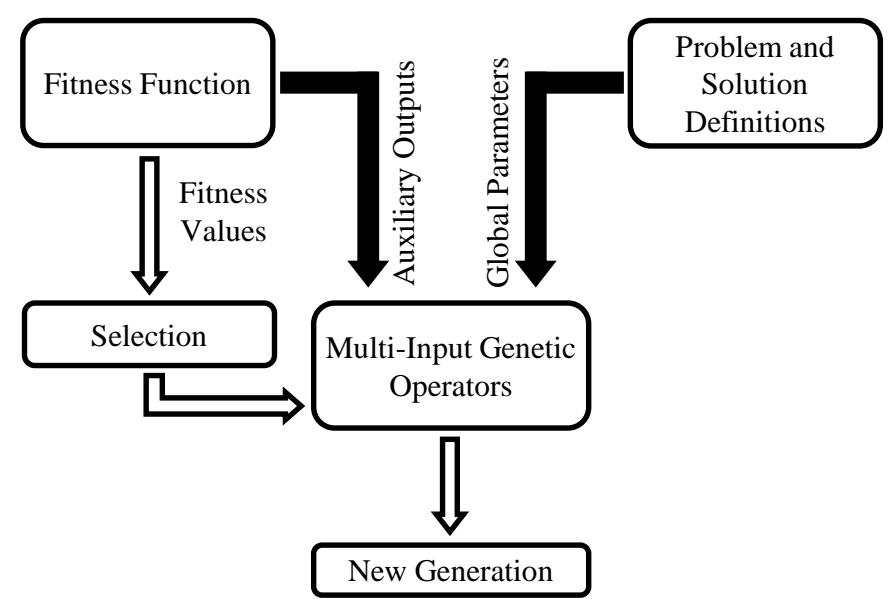

Figure 1: Schematic view of $A M I G O$. The highlighted arrows indicate the novel elements of the framework. The use of additional information in the genetic operators enables a much faster convergence to the optimal solution. 


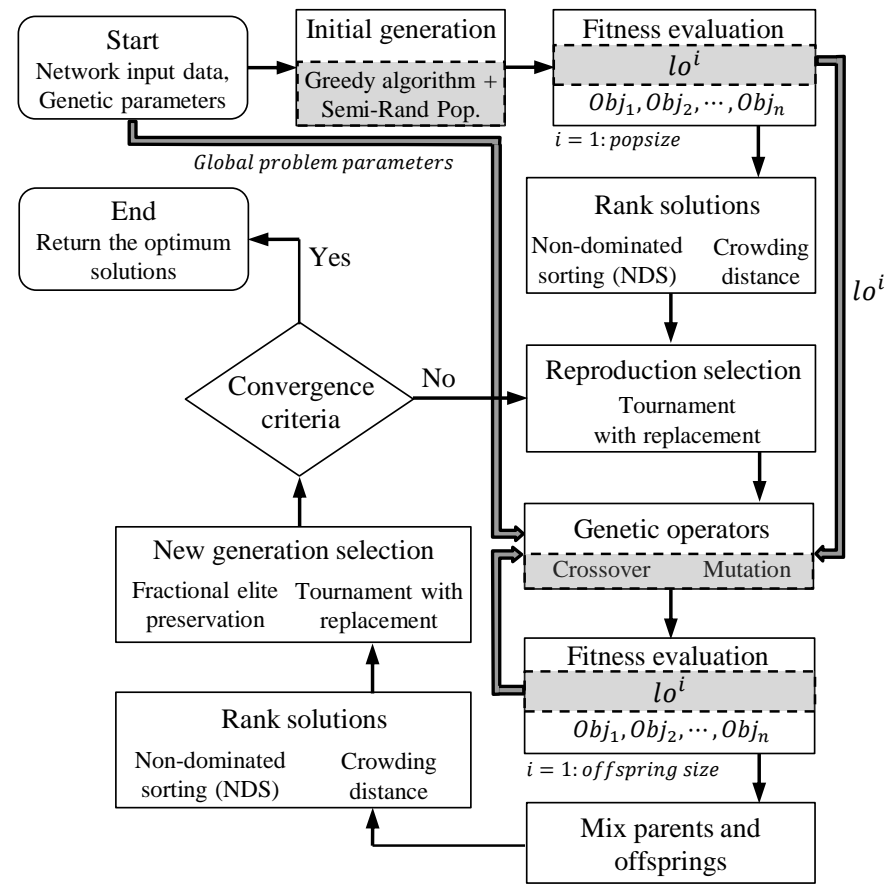

Figure 2: Flowchart of $A M I G O$ for multi-objective optimization. The highlighted sections and arrows indicate the new aspects of the algorithm. Note that the two selection processes shown in the figure are different and satisfy different purposes: the New Generation Selection is carried out to reduce the size of the mixed population (equal to $2 \cdot$ popsize) to the original popsize, then the Reproduction Selection is performed to choose parents used in reproduction functions (i.e. mutation and crossover). 
(a)

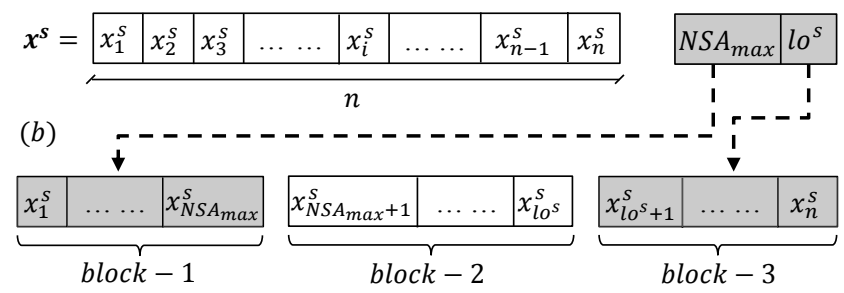

Figure 3: Trial solution representation 


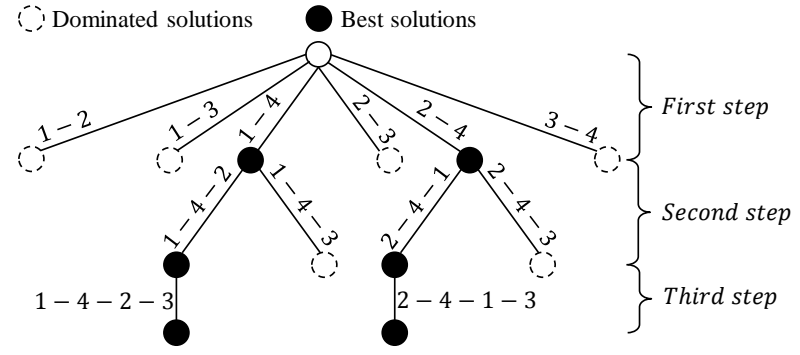

Figure 4: Greedy algorithm optimization example for a 4-activity project and $N S A_{\max }=2$. 


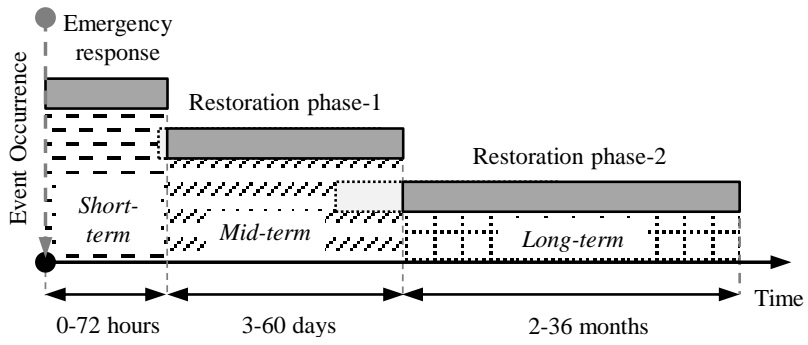

Figure 5: Disaster management phases 


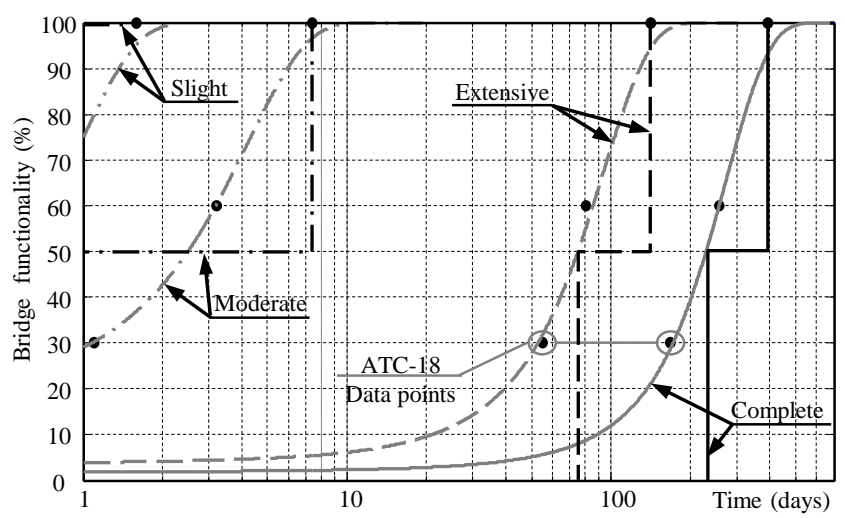

Figure 6: Restoration model for carrying bridges: fitted normal CDF (gray curves), stepwise restoration curves used in this study (black lines). 


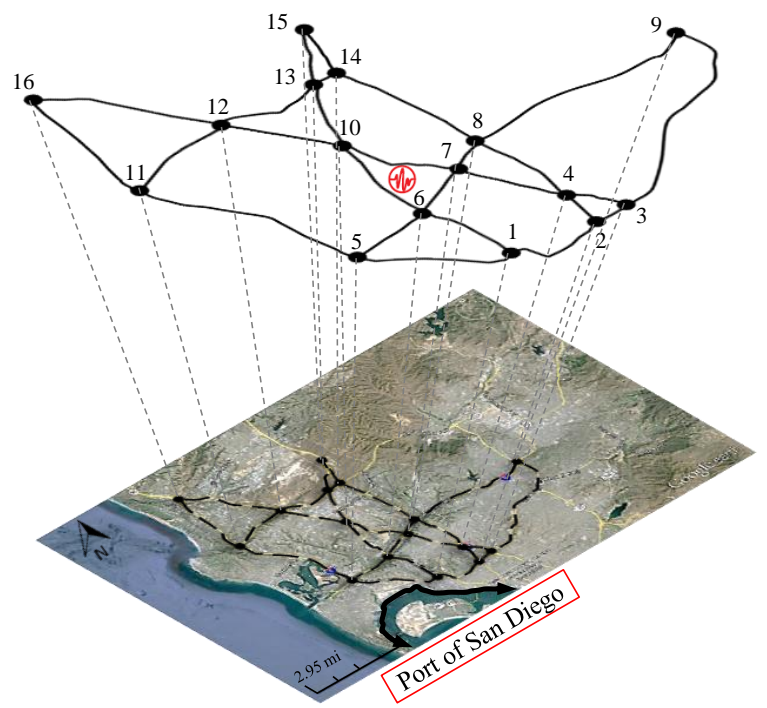

Figure 7: Port of San Diego and the modeled transportation network 


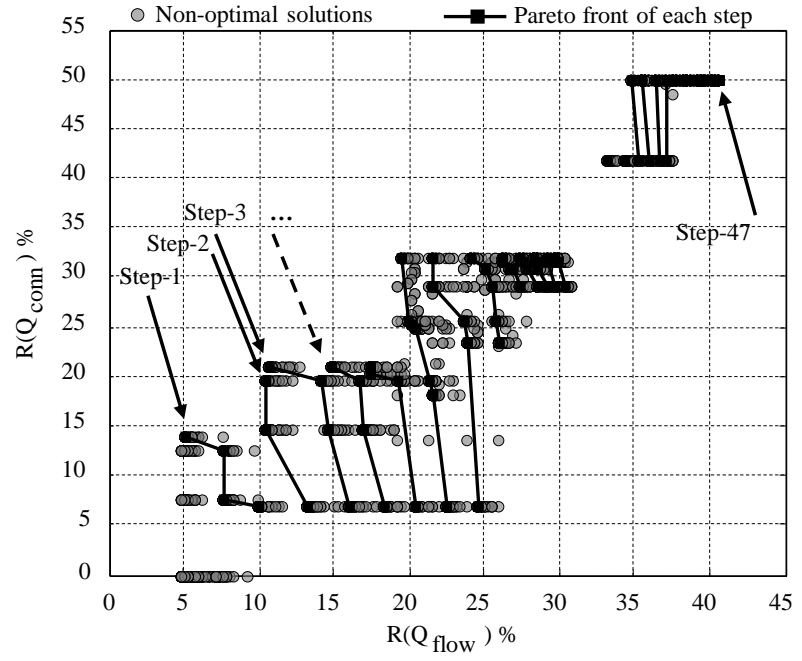

Figure 8: Greedy algorithm preliminary optimization 

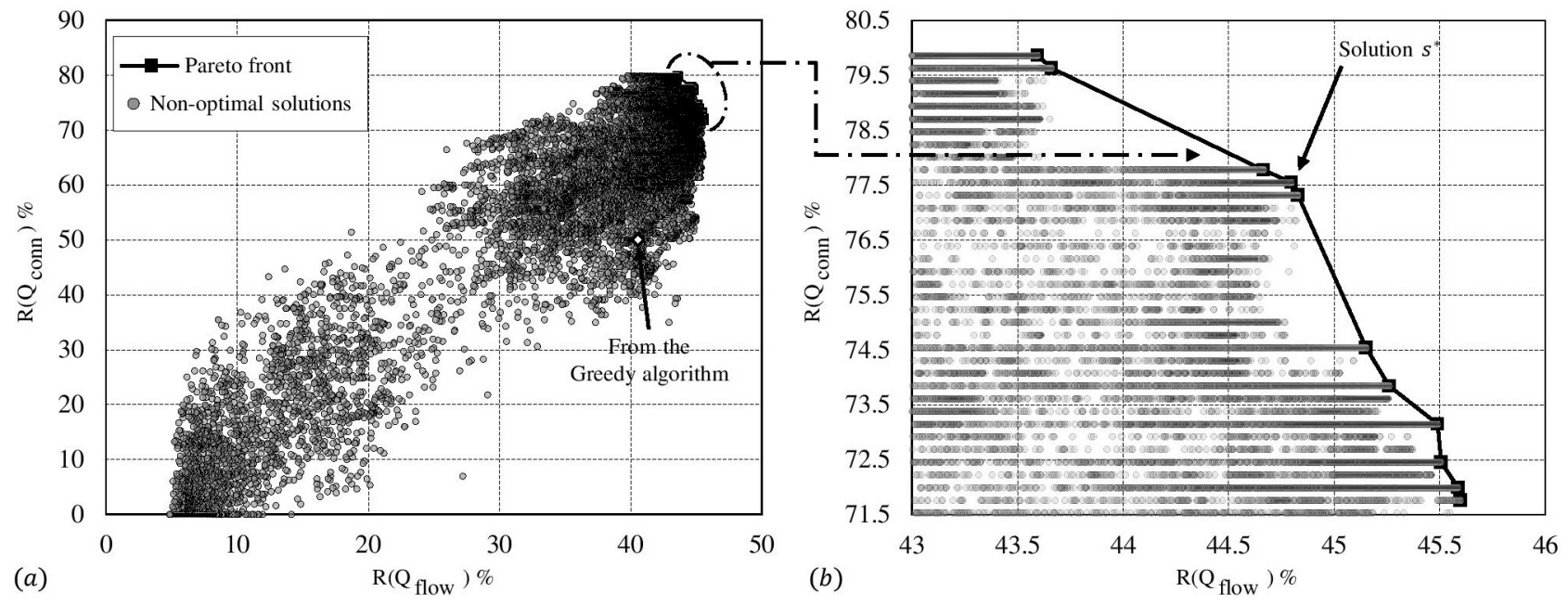

Figure 9: $A M I G O$ optimization results: (a) Trial solutions, (b) Pareto-front 


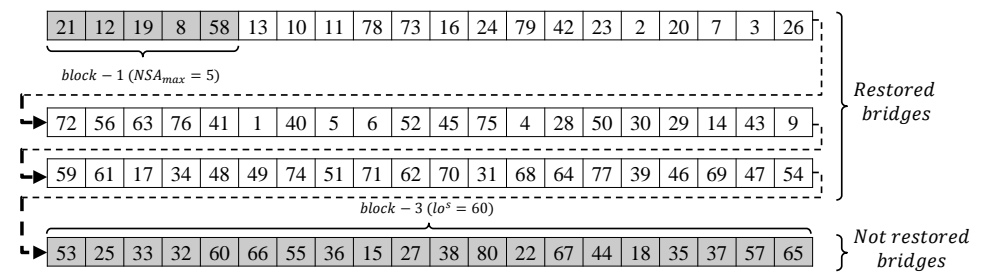

Figure 10: Solution $s^{*}$ (numbers in the sequence are bridge numbers as shown in Table 2) 


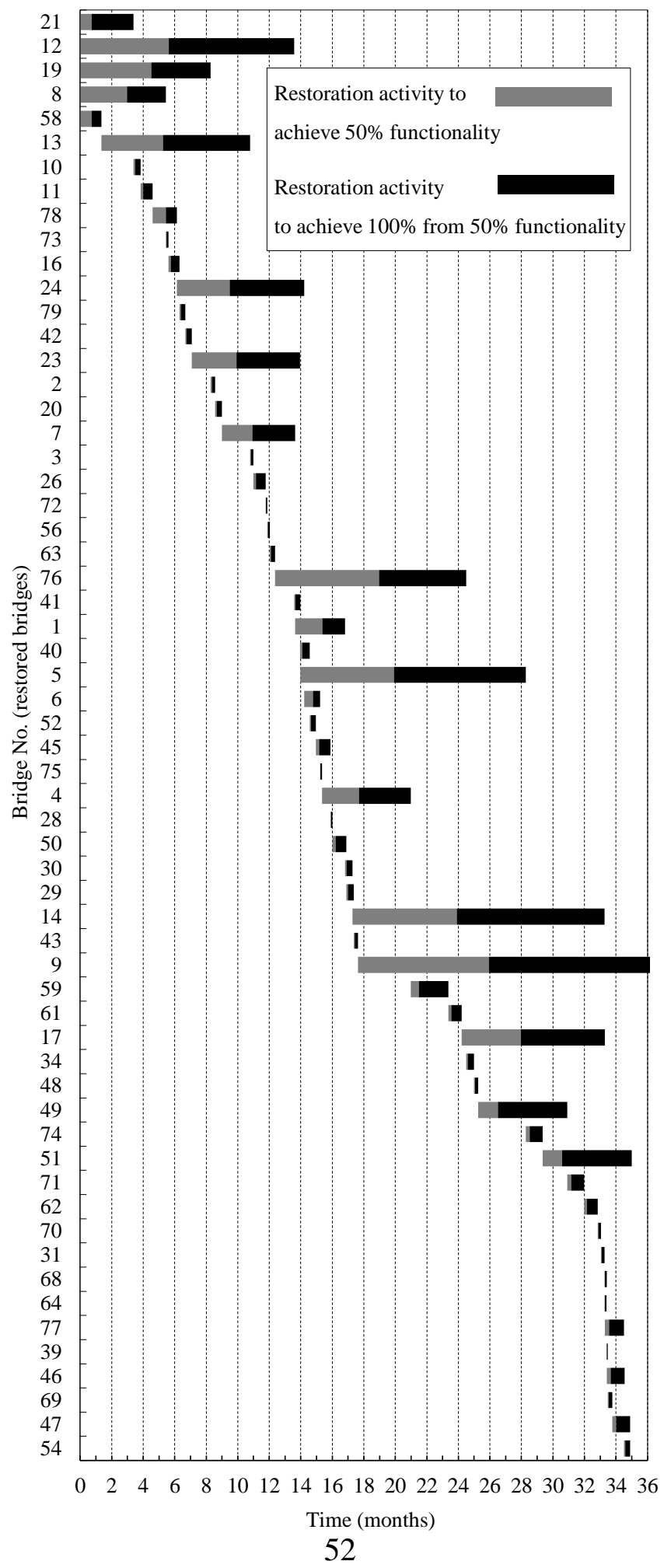

Figure 11: Gantt chart for solution $s^{*}$ 

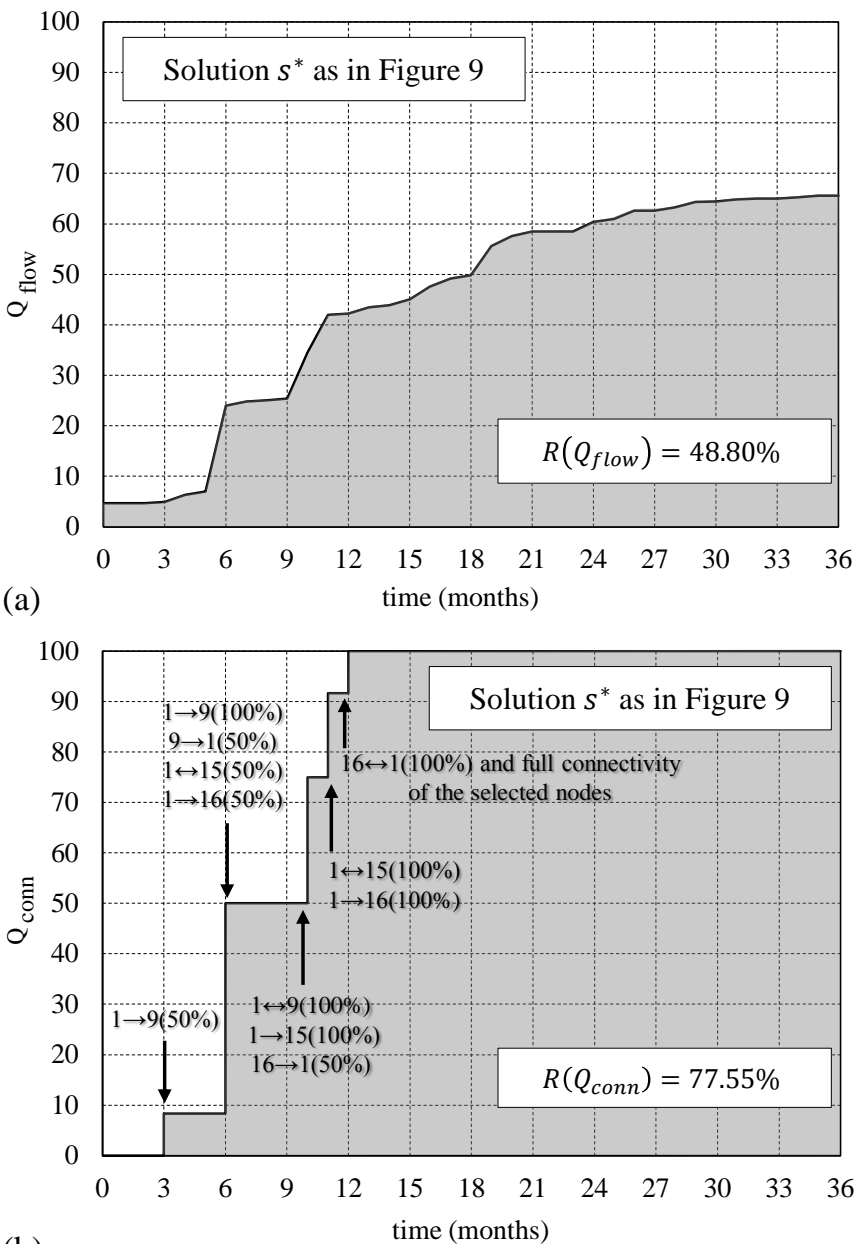

(b)

Figure 12: Evolution of the two functionality indicators for solution $s^{*}$ : (a) $Q_{f l o w}(t)$, (b) $Q_{\text {conn }}(t)$ 


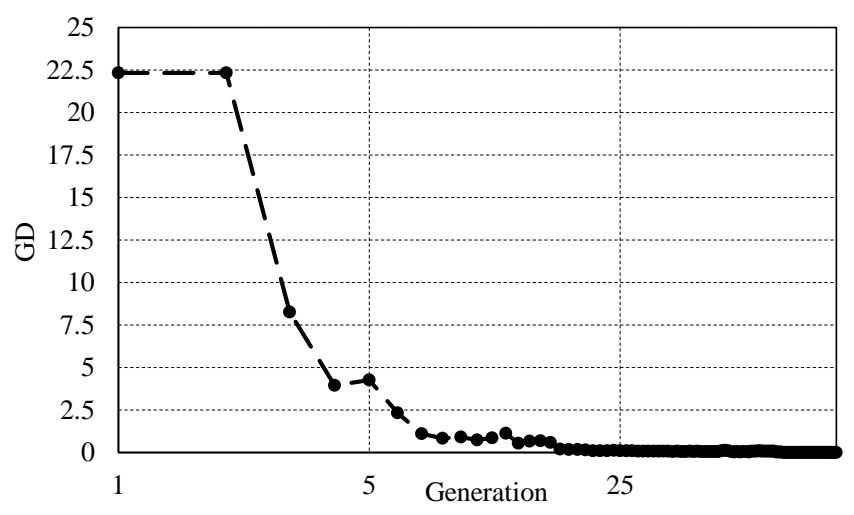

Figure 13: Evolution of the GD indicator 


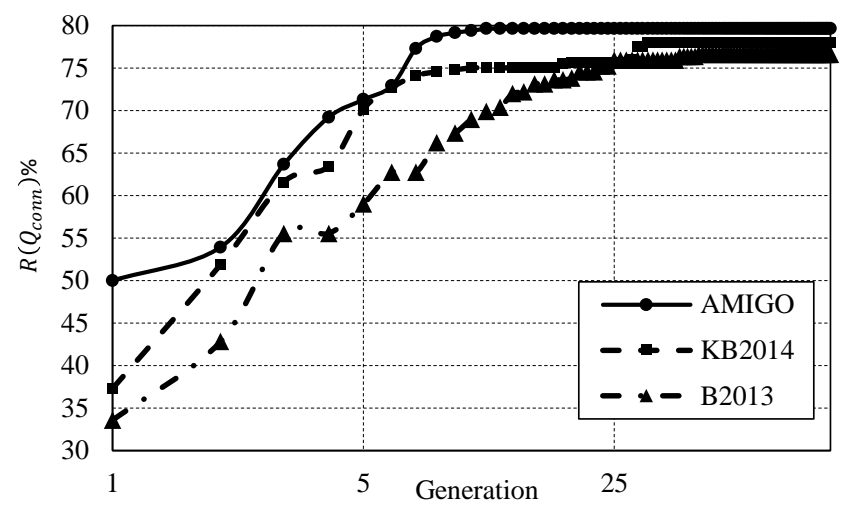

Figure 14: Best fitness values at each generation for three methodologies 